\title{
Current status of rice brown planthopper against insecticides in China (Abstract)
}

\author{
Li Jianhong \\ College of Plant Science and Technology, Huazhong Agricultural University, Wuhan, China \\ Corresponding author: jianhl@ mail.hzau.edu.cn
}

\begin{abstract}
The brown planthopper (Nilaparvata lugens Stål) is one of the most destructive pest of rice crops throughout Asia, and this pest has developed high resistance to many groups of insecticides following their heavy and frequent application. In order to investigate the current status of resistance to frequently used insecticides, a total of fifty-three field populations of $N$. lugens were collected in nine Chinese provinces, and resistance to insecticides was monitored from 2013 to 2017. The result showed that field populations of $N$. lugens had developed susceptible to low resistance levels to sulfoxaflor (RR 0.8-8.0), susceptible to moderate resistance levels to etofenprox (RR 1.0-11.2), triflumezopyrim (RR 1.2-17.1) and nitenpyram (RR 1.2-17.3), low to moderate resistance levels to chlorpyrifos (RR 7.7-57.4), low to high resistance levels to clothianidin (RR 6.1-138.8) and dinotefuran (RR 6.4-187.0), and moderate to high resistance levels to isoprocarb (RR 15.4-113.9) and thiamethoxam (RR 25.9-1025.6). Furthermore, all fifty-three field populations of $N$. lugens had developed high resistance levels to imidacloprid (RR 220.4-6324.0) and buprofezin (RR 306.4-11573.2). Except etofenprox, nitenpyram and chlorpyrifos, the resistance ratio of $N$. lugens to other insecticides all showed an increasing trend from 2013 to 2017. The obvious increase in resistance to widely applied insecticides indicates that insecticide resistance management strategies are urgently needed to prevent or delay further increase of insecticide resistance in N. lugens.
\end{abstract}

Keywords: Brown planthopper, insecticides, China. 
